\title{
Sinus of Valsalva Sclerosis is Associated with Conduction Abnormalities on 12-Lead Standard ECG
}

Keywords: Sinus of valsalva sclerosis; Atrioventricular block; Conduction abnormality

\begin{abstract}
Background: Sclerosis of the sinus of valsalva correlates with cardiovascular disease burden. The anatomic proximity of the aortic root to the conduction system suggests potential association between sinus of valsalva sclerosis and conduction abnormalities.

Methods: ECGs and clinical records from 129 consecutive patients undergoing transesophageal echocardiography for routine stroke work-up were collected from a single centre database. TEE standard loops and stand-ard 12 lead ECG recordings were analysed by 3 experienced cardiologists.

Results: Of 129 patients, sclerosis of the sinus of valsalva was detected in $72(55.8 \%), 39(30.2 \%)$ had aortic valve sclerosis and 67 $(51.9 \%)$ showed plaques of at least grade I in the ascending aorta. Conduction abnormalities and arrhythmias were found in 63 patients amongst others 30 with grade I, or higher AV-block, 10 patients with sinus node dysfunction, and 20 with atrial fibrillation. Sclerosis of the sinus of valsalva occurred in 42 of these 63 patients $(p=0.022)$. Of 30 patients with $\mathrm{AV}$-block grade I or higher, 26 presented a sinus valsalva sclerosis $(p<0.001), 7$ of 10 patients with sinus node dysfunction presented a sinus valsalva sclerosis $(p=0.508)$ and 15 of 20 with atria fibrillation ( $p=0.081)$.
\end{abstract}

Conclusion: Sclerosis of the sinus of valsalva is associated with atrioventricular conduction abnormalities.

\section{Background}

Transesophageal echocardiography (TEE) allows precise evaluation of left atrial anatomy, including the auriculum as well as left ventricular output tract (LVOT), mitral valve, aortic valve and ascending aorta [1]. Because of its sensitivity for detection of thromboembolic sources in the left heart, TEE has become an important tool for the evaluation of patients with stroke or transient ischemic attack.

Patients with cardiac arrhythmias of different aetiologies often show calcifications of the aortic valve [2], the LVOT, the sinus of Valsalva [3] or of the aorta [4]. Particularly, aortic valve sclerosis is frequently found in elderly people, and is associated with atrial flutter, left atrium enlargement and diastolic dysfunction [5]. Sinus of valsalva, dilation occurs in many patients with heart diseases, and its sclerosis predicts severe atherosclerosis of the aorta $[3,6]$. There are, however, no data regarding a correlation between conduction abnormalities and sinus of valsalva sclerosis yet. This is somehow surprising, as cardiologists who frequently perform TEEs are aware of higher arrhythmia prevalence in patients with aortic sclerosis [7]. Moreover, patients with heavy annular calcifications around the

\section{Journal of}

Cardiobiology

\section{Susanne Markendorf ${ }^{1}$, Tobias Fuchs ${ }^{1}$, Dominik Mueller ${ }^{1}$, Heiner Vontobel, Urs Eriksson ${ }^{1,3}$, and Nazmi Krasniqi ${ }^{12^{*}}$}

${ }^{\prime}$ Division of Cardiology, GZO - Zurich Regional Health Center, Wetzikon, Switzerland

${ }^{2}$ Cardiology, Cardiovascular Center, University Hospital Zurich, Switzerland

${ }^{3}$ Cardioimmunology, Center of Molecular Cardiology, University of Zurich, Switzerland

\section{*Address for Correspondence}

Nazmi Krasniqi, MD, Division of Cardiology, GZO - Zurich Regional Health Center, Spitalstrasse 66, CH-8620 Wetzikon, Switzerland, Tel: ++41-44934-2352; Fax: ++41-44-934-1199; E-mail: nazmi.krasniqi@gzo.ch

Submission: 07 July, 2015

Accepted: 18 December, 2015

Published: 23 December, 2015

Copyright: (๑) 2015 Markendorf S et al. This is an open access article distributed under the Creative Commons Attribution License, which permits unrestricted use, distribution, and reproduction in any medium, provided the original work is properly cited.

Reviewed \& Approved by: Dr. MacArthur A Elayda, Department of Cardiovascular Epidemiology and Medical Infor-matics, Texas Heart Institute, USA

atrioventricular sinus area who underwent aortic valve replacement surgery are significantly more likely to need temporary cardiac pacing than those without annular calcification [8].

We analysed TEE loops and standard 12-lead ECGs of 129 consecutive patients, undergoing cardiologic work-up after TIA/ stroke, for sclerosis of the sinus of Valsalva and the presence of conduction abnormalities such as atrioventricular blockage (AVB), right bundle branch blockage (RBB), left bundle branch blockage (LBB) and sinus node disease (SND).

\section{Materials and Methods}

\section{Patient population and patient baseline characteristics}

We retrospectively analysed TEE studies and standard 12-lead ECGs of 129 consecutive patients at the Cardiology division of a tertiary care regional hospital in the greater Zurich area. Patient records were analysed for demographics, co-morbidities at or before the examination date, cardiovascular risk factors, and cardiovasculardrug therapy. Patients undergoing TEE for endocarditis evaluation or with congenital heart disease were excluded. The cantonal ethics committee of Zurich acknowledged retrospective data analysis for research purposes (KEK-ZH-Nr. 2014-0529).

To characterize the study population and to identify a potential correlation between arrhythmias and sinus valsalva sclerosis, the following parameters were tabled as baseline characteristics: Age, BMI, heart disease divided in coronary heart disease (1-, 2- or 3- vessel), dilated cardiomyopathy and valvular cardiomyopathy, dyslipidaemia, smoking, type II diabetes mellitus, hypertension, history of ST-elevation myocardial infarction, cerebrovascular insult, transient ischemic attack, peripheral arterial occlusive disease, 
Citation: Markendorf S, Fuchs T, Mueller D, Vontobel H, Eriksson U, et al. Sinus of Valsalva Sclerosis is Associated with Conduction Abnormalities on 12-Lead Standard ECG. J Cardiobiol. 2015;3(1): 5.

ISSN: 2332-3671

sclerosis of the aorta, ICD or permanent pacemaker device, and medications.

\section{Transesophageal echocardiography}

Transoesophageal echocardiography was performed according to the guidelines of the American heart association [9] and executed with Siemens Acuson SC2000, Z6Ms transducer. All procedures were digitally stored. Each loop was recorded over 3 ECG gated cycles and analysed independently by three experienced cardiologists. Sclerosis of the sinus of valsalva was defined as a hyperechogenic pattern of the sinus of valsalva with an acoustic shadow. Aortic valve sclerosis was defined as focal areas of valve thickening, typically located in the leaflet center with commissural sparing and normal leaflet mobility and evidence for impaired motility of at least 1 cusp with flow velocity below $2.5 \mathrm{~m} / \mathrm{s}$ [10]. Plaques in the aorta were notified and classified according to the classification of Montgomery [11].

\section{ECG analysis}

All 12-lead standard ECGs were recorded with a Schiller Cardiograph model AT-10 Plus, at a paper speed of $25 \mathrm{~mm} / \mathrm{s}$. In our hospital we obtain standard ECG (10 electrodes) using the traditional configuration with the limb lead electrodes placed on the distal limbs. Conduction abnormalities were defined as, AV-block grade I or higher, LBB partial or complete, RBB partial or complete, sinus node dysfunction (refers to sinus bradycardia, chronotropic incompetence and/or sinus pause).

\section{Statistical analysis}

Quantitative variables were expressed as mean \pm SD and categorical variables as frequencies or percentages. Different groups were compared with Chi-square tests, and exact fisher test, significance of correlation between two binary variables was checked with Pearson test of correlation, appropriate using SPSS 21.0 statistical software package (SPESS, Chicago, IL, USA). P values of $<0.05$ were considered statistically significant.

\section{Results}

Patient baseline characteristics are shown in Tables 1 and 2, 72 patients had sclerosis of the sinus of valsalva, 39 had aortic valve sclerosis. 30 of those had as well sinus valsalva sclerosis as aortic valve sclerosis. 67 patients showed plaques grades I-IV in the ascending aorta (see Table 3). Of 63 patients with arrhythmia or conduction abnormality, 30 had grade I or higher AVB (AVB grade 1: 22, AVB grade 2: 5, AVB grade 3: 3), 10 had SND, 27 showed LBB, and 12 RBB. 20 patients had atrial fibrillation and 6 atrial flutter. About one half of the patients had a history of cardiovascular pathology including coronary artery disease (27), valvular disease (22), dilated cardiomyopathy (1), known cerebrovascular disease (64), and

Table 1: Baseline characteristics mean +/- SD.

\begin{tabular}{|l|c|c|}
\hline \multicolumn{1}{|c|}{ Baseline Characteristics } & Mean & Range \\
\hline Age at time of TEE $(\mathrm{y})$ & $66.29 \pm 12.16$ & $38-91$ \\
BMI $\left(\mathrm{kg} / \mathrm{m}^{2}\right)$ & $26.73 \pm 3.52$ & $20.1-35$ \\
\hline Calcium value $(\mathrm{mmol} / \mathrm{L})$ & $2.36 \pm 0.42$ & $1.9-5.7$ \\
\hline Cholesterine $(\mathrm{mmol} / \mathrm{L})$ & $4.81 \pm 1.25$ & $2-7.8$ \\
\hline LDL $(\mathrm{mmol} / \mathrm{L})$ & $2.97 \pm 1.16$ & $0.77-5.77$ \\
\hline Packyears & $13.28 \pm 20.19$ & $0-80$ \\
\hline
\end{tabular}

Table 2: Characteristics in number and percentage of the study population.

\begin{tabular}{|l|c|c|}
\hline Total 129 patients & N & $\%$ \\
\hline Male sex & 82 & 63.6 \\
\hline Dyslipidemia & 66 & 51.2 \\
\hline Smoking & 57 & 44.2 \\
\hline Type II Diabetes mellitus & 22 & 17.1 \\
\hline Hypertonia & 69 & 53.5 \\
\hline Coronary heart disease & 27 & 20.9 \\
\hline Valvular cardiomyopathy & 22 & 17.1 \\
\hline Dilatative cardiomyopathy & 1 & 0.8 \\
\hline History of STEMI & 14 & 10.9 \\
\hline Cerebrovascular insult & 64 & 49.6 \\
\hline Aortic dissection & 3 & 2.3 \\
\hline Peripheral arterial occlusal disease & 23 & 17.8 \\
\hline Atherosclerosis & 39 & 30.2 \\
\hline Permanent pacemaker & 3 & 2.3 \\
\hline
\end{tabular}

Table 3: Appearance of sclerosis in the aortic valve and the sinus of valsalva and of aortic plaques divided into the four Montgomery stages in all subjects.

\begin{tabular}{|c|c|c|c|}
\hline & & $\mathbf{N}$ & $\%$ \\
\hline \multicolumn{2}{|c|}{ Aortic valve sclerosis } & 39 & 30.2 \\
\hline \multicolumn{2}{|c|}{ Sclerosis of Sinus of Valsalva } & 72 & 55.8 \\
\hline \multicolumn{2}{|l|}{ Aortic plaque } & 67 & 51.9 \\
\hline- & Montgomery I & 11 & 5.8 \\
\hline - & Montgomery II & 27 & 20.9 \\
\hline- & Montgomery III & 13 & 10.1 \\
\hline- & Montgomery IV & 16 & 12.4 \\
\hline
\end{tabular}

peripheral arterial occlusive disease (23). 3 patients had pacemakers. Table 4 summarizes medical treatments of the cohort.

Out of 63 Patients with arrhythmia, 42 presented a sinus valsalva sclerosis 21 did not and only 18 showed an aortic valve sclerosis. Of the 66 patients without arrhythmia 30 presented a sinus valsalva sclerosis ( $\mathrm{p}=0.021$, correlation coefficient 0.214$)$ and 21 an aortic valve sclerosis $(\mathrm{p}=0.500) .43$ patients showed disorders of the cardiac conduction system such as AVB, RBB, LBB and SND. 28 of those patients showed sinus of valsalva sclerosis $(\mathrm{p}=0.177) .18$ of them showed aortic valve sclerosis $(\mathrm{p}=0.097)$. Of the patients without AVB, RBB, LBB or SND $(\mathrm{N}=86) 44$ showed a sinus valsalva sclerosis and 21 an aortic sclerosis. Out of the 30 patients with AV-blockage grade I or higher, 26 had a sclerosis of the sinus of valsalva $(\mathrm{p}=0.000$, correlation coefficient 0.342 ), 11 patients presented an aortic valve sclerosis ( $\mathrm{p}=0.642$ ). To exclude the possibility of iatrogenic AV-blockages, betablocking medication and calcium-channel-inhibitors were checked for this group: Only 4 of the patients with AV-blockage took a betablocker, 3 a calcium-channel-blocker. We therewith exclude that AV-node decelerating medication biased our results. 16 of 27 patients with left bundle branch blockage present a sclerosis of the sinus of valsalva, which is not significant $(\mathrm{p}=0.829) .7$ of 10 patients with sinus node disease show a sclerosis of the sinus of valsalva what as well is not significant ( $\mathrm{p}=0.508)$ and only 3 showed an aortic valve sclerosis. 6 of 12 patients with right bundle branch blockage have a sclerosis of the 
Table 4: Medical treatment of all subjects.

\begin{tabular}{|l|c|c|}
\hline Medication & N & $\%$ \\
\hline Betablockers & 31 & 26.3 \\
\hline Alphablockers & 5 & 3.9 \\
\hline Calcium channel blocker & 13 & 10.1 \\
\hline RAAS inhibitor & 38 & 30.2 \\
\hline Diuretics & 32 & 24.0 \\
\hline Vasodilatators & 5 & 3.9 \\
\hline Antiarrhythmics & 0 & 0 \\
\hline Anticoagulants & 73 & 56.6 \\
\hline Statins & 64 & 49.6 \\
\hline Antiosteoporotics & 13 & 10.1 \\
\hline Steroids & 5 & 3.9 \\
\hline
\end{tabular}

sinus of valsalva what is not significant $(\mathrm{p}=0.764)$, whereby 7 showed a sclerosis of the aortic valve, what as well is not significant $(\mathrm{p}=0.075)$ 15 of 20 patients with atrial fibrillation showed a sinus sclerosis in the echocardiography, what is not significant $(\mathrm{p}=0.081)$ only 7 presented an aortic valve sclerosis $(\mathrm{p}=0.480)$.

27 of the 39 patients with atherosclerosis showed a sinus sclerosis as well, what is a significant result $(\mathrm{p}=0.043$, correlation coefficient 0.178 ), only 13 presented a sclerosis of the aortic valve what is not significant ( $\mathrm{p}=0.656) .57$ of 67 patients with plaques in the aorta ascendens present a sinus sclerosis what is highly significant ( $\mathrm{p}<0.001$, correlation coefficient 0.640 ), only 25 of the patients with aortic valve sclerosis showed plaques in the aorta ascendes $(\mathrm{p}=0.965)$. 29 of 39 patients with aortic valve sclerosis show a sinus sclerosis as well ( $\mathrm{p}=0.015$, correlation coefficient 0.250 ). All other correlations between sinus sclerosis and the different parameters showed no significant results as visible in Table 5 .

\section{Discussion}

In this study we describe for the first time a correlation between calcification of the sinus of valsalva and the presence of conduction system abnormalities and arrhythmia. Of particular interest is the significant correlation between sclerosis of the sinus of valsalva and AVB despite the rather small study population and the fact, that this was independent of aortic valve sclerosis. With a correlation coefficient of 0.342 we can even show a medium correlation of these two parameters what can be seen as great advantage in a retrospective trial with a relatively small study population. Drugs like calcium-channel inhibitors, betablockers and digoxin are known to be related with cardiac conduction abnormalities, but mostly, these abnormalities are not directly induced by drugs [12]. Anyway importantly, only 7 of the 30 patients with AVB were treated with betablockers or calcium-channel inhibitors and none with digitalis. In the population without AVB, 30 patients were treated with betablocker and 10 with calcium-channel blockers.

As other risk factors for AVB coronary heart disease and myocardial infarction are known [13], although the incidence of high grade AVB in patients with myocardial infarction is reduced since patients are treated with primary percutaneous intervention [14]. In this study, incidence of patients after myocardial infarction in the population with AVB is 3 of 30 , incidence of patients with coronary heart disease is 6 of 30 . We therewith can neglect that this is a major trigger for AVB in our population. However so far, no other study correlated sclerosis of the sinus of valsalva with arrhythmias and/or conduction abnormalities. There is indirect evidence from patients undergoing transcatheter aortic valve replacement that a thickened and calcified aortic root predicts AV conduction abnormalities on follow-up $[15,16]$. Similarly, a high incidence of left bundle branch blockage was found after TAVI implantation [17]. In the context of transcatheter aortic intervention, however, the procedure requires the expansion of prosthetic devices, which might directly affect integrity of the adjacent conduction system in patients with aortic root calcification [18]. Accordingly, prosthesis depth and size were independent predictors for the occurrence of a new left bundle branch block. Moreover, it is not surprising that aortic root sclerosis is predictive for conduction abnormalities in TAVI. The relevance of sinus of valsalva sclerosis, however, has not been evaluated yet in this context.

In our cohort, analysis of conduction abnormalities also pointed to an association between sclerosis of sinus of valsalva and atrial fibrillation. These observations, however, are more difficult to interpret. The question therefore arises, whether sinus of valsalva sclerosis represents readout for structural atrial remodelling affecting the cardiac conduction system and electric activation in general. This is a striking hypothesis, but larger and prospective studies are certainly warranted to specifically address this question.

Sclerosis of the sinus of valsalva had been recognized as predictor for atherosclerosis. We could show a significant association between those two parameters nevertheless the correlation coefficient points only to a low correlation between them. This might be due to our rather small study population. Moreover, an inverse association between lumbar bone mineral density and atherosclerotic arterial calcium could be identified $[3,19]$. Interestingly, osteoporosis and

Table 5: Correlation between Sinus Valsalva Sclerosis/Aortic Valve Sclerosis and AV-conduction Abnormalities/Arrhythmias.

\begin{tabular}{|l|c|c|c|c|c|}
\hline \multicolumn{1}{|c|}{ Results } & N & $\begin{array}{c}\text { Sclerosis of } \\
\text { Sinus of } \\
\text { Valsalva }\end{array}$ & $\mathbf{p}$ & $\begin{array}{c}\text { Aorticvalve } \\
\text { sclerosis }\end{array}$ & $\mathbf{p}$ \\
\hline No arrhythmia & 66 & 30 & 0.022 & 21 & 0.500 \\
\hline Arrhythmia & 63 & 42 & 0.022 & 18 & 0.500 \\
\hline $\begin{array}{l}\text { AVB, RBBB, LBBB } \\
\text { or SND }\end{array}$ & 43 & 28 & 0.177 & 18 & 0.097 \\
\hline $\begin{array}{l}\text { No AVB, RBBB, } \\
\text { LBBB or SND }\end{array}$ & 86 & 44 & 0.177 & 21 & 0.097 \\
\hline AVB I, II \& III & 30 & 26 & $<0.001$ & 11 & 0.642 \\
\hline SND & 10 & 7 & 0.508 & 3 & 0.906 \\
\hline RBBB & 12 & 6 & 0.764 & 7 & 0.075 \\
\hline LBBB & 27 & 16 & 0.829 & 12 & 0.180 \\
\hline Atrial fibrillation & 20 & 15 & 0.081 & 7 & 0.480 \\
\hline Arial flutter & 6 & 3 & 1.000 & 1 & 0.330 \\
\hline Atherosclerosis & 39 & 27 & 0.043 & 13 & 0.656 \\
\hline Diabetes & 22 & 16 & 0.095 & 9 & 0.288 \\
\hline Hypertension & 69 & 38 & 0.861 & 21 & 0.216 \\
\hline
\end{tabular}




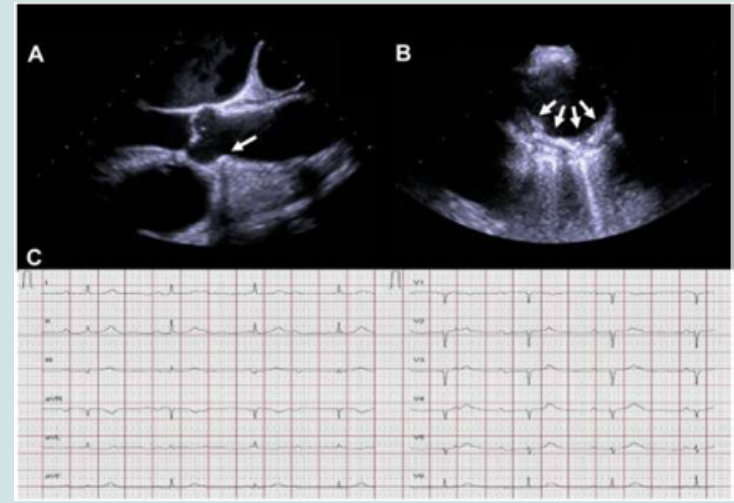

Figure 1: Images of transoesophageal echogardiography of a 78 year old female patient with AV-Block $(C)$ demonstrating a calcified plaque of the sinus of valsalva ( $A$; white arrow) as well as diffuse plaques montgomery $V$ in the descending aorta (B; white arrows).

atherosclerosis often coexist age-independently even in relation to age matched controls [20]. In fact, lower bone density was significantly associated with calcified atherosclerosis on multivariable adjustment, even in some cases where traditional risk factors as hypertension and cholesterol were not. Consequently, these observations argue for a common pathogenic mechanism involving endothelial dysfunction and oxidized lipid metabolism $[21,22]$. Such a pathogenic mechanism might well affect various cardiac structures including the conduction system. Further studies are needed to delineate a possible link between sinus of valsalva sclerosis, osteoporosis, and tachy- or brady arrhythmia.

Our study has several limitations. Its retrospective design is prone to selection bias. Given the fact, that almost all patients undergoing TEE had a history of stroke or TIA, prevalence of both, sclerosis of sinus of valsalva as well as atrial fibrillation is much higher in the study population than in age matched healthy subjects. On the other hand, retrospective analysis of standard ECGs also underestimates the true prevalence of conduction abnormalities, as well as its incidence in patients with sclerosis of the sinus of valsalva. Furthermore, for getting significant results despite the relatively small study population, patients with sinus valsalva sclerosis and aortic valve sclerosis were not classified mutually exclusive. This however would be necessary for prospective studies with larger study populations to clearly address the predictive impact of sinus of valsalva sclerosis as risk factor for arrhythmias and/or conduction abnormalities in the future. In such studies, sinus of valsalva sclerosis and aortic valve sclerosis should be analysed mutually exclusive and all other risk factors of cardiac conduction abnormalities have to be evaluated as well. Anyway that might be ethnically difficult as TEE is an invasive diagnostic method.

Despite these limitations, our data suggests a not yet described role for sinus of valsalva sclerosis as an indicator for disorders of cardiac conduction system.

In conclusion, sclerosis of the sinus of valsalva is associated with high prevalence of arrhythmias such as atrial fibrillation. Based on our data, we cannot exclude that other risk factors are causally linked to the described arrhythmias. However in this study we were able to show a correlation between sclerosis of sinus of valsalva and atrioventricular-conduction abnormalities.

\section{References}

1. American College of Cardiology Foundation Appropriate Use Criteria Task Force, American Society of Echocardiography, American Heart Association, American Society of Nuclear Cardiology, Heart Failure Society of America, et al. (2011) ACCF/ASE/AHA/ASNC/HFSA/HRS/SCAI/SCCM/SCCT/SCMR 2011 appropriate use criteria for echocardiography. A report of the American College of Cardiology Foundation appropriate use criteria task force, American Society of Echocardiography, American Heart Association, American Society of Nuclear Cardiology, Heart Failure Society of America, Heart Rhythm Society, Society for Cardiovascular Angiography and Interventions, Society of Critical Care Medicine, Society of Cardiovascular Computed Tomography, Society for Cardiovascular Magnetic Resonance American College of Chest Physicians. J Am Soc Echocardiogr 24: 229-267.

2. Sainger R, Grau JB, Branchetti E, Poggio P, Lai E, et al. (2013) Comparison of transesophageal echocardiographic analysis and circulating biomarker expression profile in calcific aortic valve disease. J Heart Valve Dis 22: 156165.

3. Finkelhor RS, Youssefi ME, Mohan SK, Bahler RC (1998) Aortic sinotubular junction calcium as a marker of severe aortic atherosclerosis. Am J Cardiol 82: 1549-1552, A8.

4. Berko BA, Owen AN, Zwas DR (2003) Echocardiography. The role of this noninvasive test in the geriatric population. Geriatrics 58: 30-34; quiz 35

5. Naito Y, Yamazaki K (2013) Preoperative left atrial volume index predicts postoperative atrial fibrillation in patients with severe aortic valve stenosis. J Anesth 27: 699-704.

6. Rabkin SW, Jue J, Tsang MY (2006) Aortic valve sclerosis is associated with an echocardiographically determined thinner aortic wall. J Heart Valve Dis 15: 158-164.

7. Palmiero $P$, Maiello M, Passantino A, Wasson S, Reddy HK (2007) Aortic valve sclerosis: is it a cardiovascular risk factor or a cardiac disease marker? Echocardiography 24: 217-221.

8. Alwaqfi NR, Ibrahim KS, Khader YS, Baker AA (2014) Predictors of temporary epicardial pacing wires use after valve surgery. J Cardiothoracic Surg 9: 33.

9. Cheitlin MD, Armstrong WF, Aurigemma GP, Beller GA, Bierman FZ, et al. (2003) ACC/AHA/ASE 2003 Guideline Update for the Clinical Application of Echocardiography: summary article. A Report of the American College of Cardiology/American Heart Association Task Force on Practice Guidelines (ACC/AHA/ASE Committee to Update the 1997 Guidelines for the Clinical Application of Echocardiography). J Am Soc Echocardiogr 16: 1091-1110.

10. Freeman RV, Otto CM (2005) Spectrum of calcific aortic valve disease: pathogenesis, disease progression, and treatment strategies. Circulation 111: 3316-3326.

11. (1996) Atherosclerotic disease of the aortic arch as a risk factor for recurrent ischemic stroke. The French Study of Aortic Plaques in Stroke Group. N Eng J Med 334: 1216-1221.

12. Zeltser D, Justo D, Halkin A, Rosso R, Ish-Shalom M, et al. (2004) Druginduced atrioventricular block: prognosis after discontinuation of the culprit drug. J Am Coll Cardiol 44: 105-108.

13. Goldberg RJ, Zevallos JC, Yarzebski J, Alpert JS, Gore JM, et al. (1992) Prognosis of acute myocardial infarction complicated by complete heart block (the Worcester Heart Attack Study). Am J Cardiol 69: 1135-1141.

14. Gang UJ, Hvelplund A, Pedersen S, Iversen A, Jøns C, et al. (2012) Highdegree atrioventricular block complicating ST-segment elevation myocardial infarction in the era of primary percutaneous coronary intervention. Europace 14: $1639-1645$.

15. Karbasi-Afshar R, Jonaidi-Jafari N, Saburi A, Khosravi A (2014) Atrioventricular block as the initial presentation of calcified bicuspid aortic valve. ARYA Atherosclerosis 10: 59-64.

16. Sekimoto Y, Nishizaki Y, Sesoko M, Sai E, Yamashita H, et al. (2014) Syncope due to paroxysmal complete atrioventricular block in a patient with aortic valve stenosis. Intern Med 53: 1347-1349.

17. Boerlage-Van Dijk K, Kooiman KM, Yong ZY, Wiegerinck EM, Damman P, 
Citation: Markendorf S, Fuchs T, Mueller D, Vontobel H, Eriksson U, et al. Sinus of Valsalva Sclerosis is Associated with Conduction Abnormalities on 12-Lead Standard ECG. J Cardiobiol. 2015;3(1): 5.

et al. (2014) Predictors and permanency of cardiac conduction disorders and necessity of pacing after transcatheter aortic valve implantation. Pacing Clin Electrophysiol 37: 1520-1529.

18. Ghadimi K, Patel PA, Gutsche JT, Sophocles A, Anwaruddin S, et al. (2013) Perioperative conduction disturbances after transcatheter aortic valve replacement. J Cardiothoracic Vasc Anesth 27: 1414-1420.

19. Hyder JA, Allison MA, Criqui MH, Wright CM (2007) Association between systemic calcified atherosclerosis and bone density. Calcif Tissue Int 80: 301 306.

20. Maichuk Elu, Voevodina IV, Mitrokhina TV, Makarova IA, lureneva SV (2014) The risk of atherosclerosis and osteoporosis development in post- ovariectomy syndrome women during hormone replacement therapy. Ter Arkh 86: 75-79.

21. Prasad M, Reriani M, Khosla S, Gossl M, Lennon R, et al. (2014) Coronary microvascular endothelial dysfunction is an independent predictor of development of osteoporosis in postmenopausal women. Vasc Health Risk Manag 10: 533-538.

22. Parhami F, Morrow AD, Balucan J, Leitinger N, Watson AD, et al. (1997) Lipid oxidation products have opposite effects on calcifying vascular cell and bone cell differentiation. A possible explanation for the paradox of arterial calcification in osteoporotic patients. Arterioscler Thromb Vasc Biol 17: 680687. 